\title{
Comparison of sonoelastography with sonourethrography and retrograde urethrography in the evaluation of male anterior urethral strictures
}

\author{
Shyam Manoharlal Talreja ${ }^{1}$, Vinay Tomar ${ }^{1}$, Sher Singh Yadav ${ }^{1}$, Usha Jaipal ${ }^{2}$, Shivam Priyadarshi ${ }^{1}$, \\ Neeraj Agarwal ${ }^{1}$, Nachiket Vyas ${ }^{1}$
}

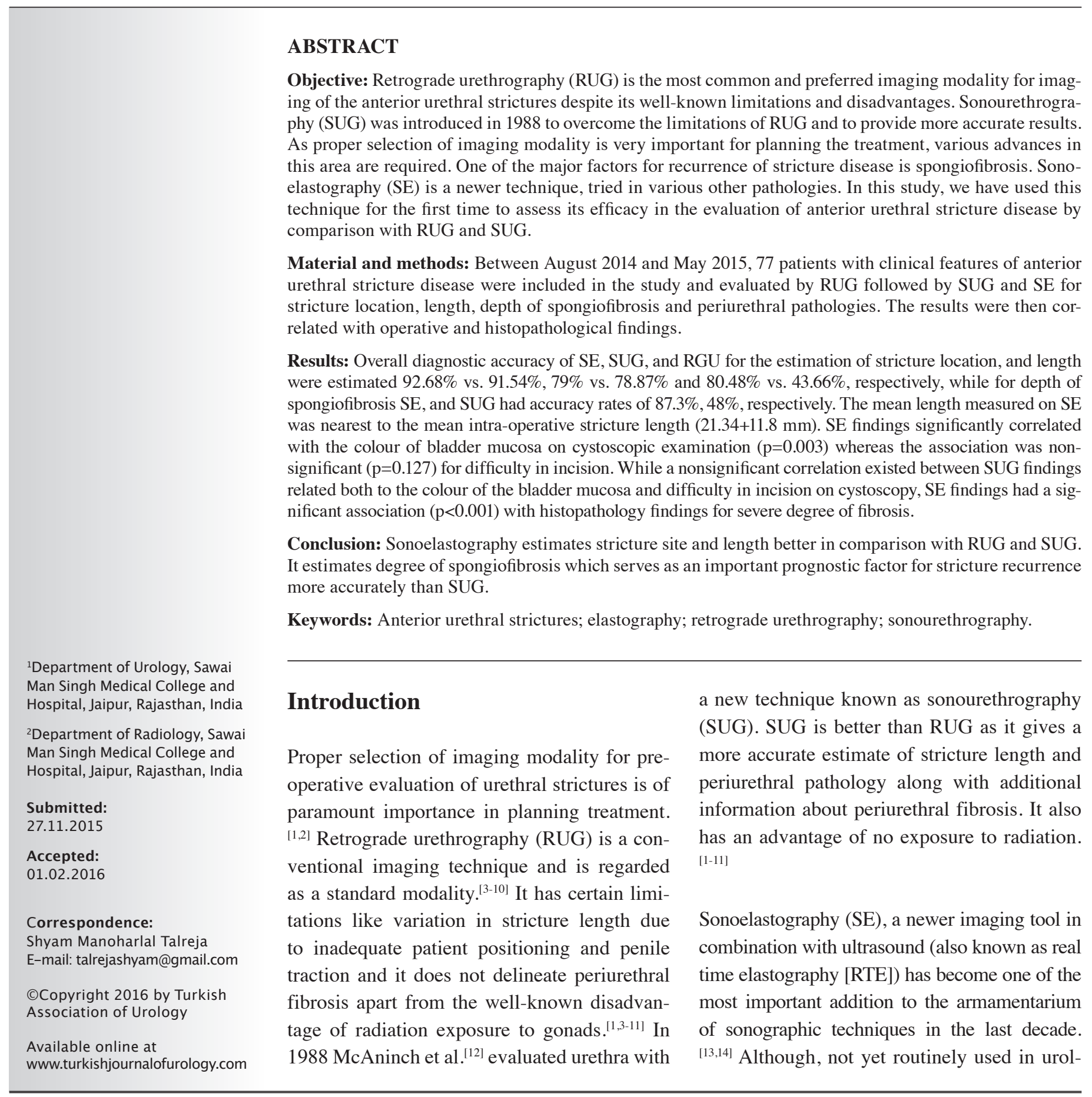


ogy clinical practice, previous studies have shown its usefulness in the differential diagnosis of breast, thyroid and prostate pathologies along with its role in diagnosing and staging acute appendicitis. ${ }^{[13-16]}$

Sonoelastography maps the elastic property of soft tissues examined ${ }^{[16]}$ which propelled us to use this technique in the evaluation of anterior urethral strictures not only for length but also for degree of spongiofibrosis and periurethral pathologies. ${ }^{[1,2,5]}$ Hence in the present study, we sought to compare the efficacy of real- time SE with SUG and RUG in the evaluation of anterior urethral strictures.

\section{Material and methods}

Present study was conducted in Department of Urology in Sawai Mann Singh Medical College and Hospital, Jaipur, Rajasthan after the approval from ethics committee of the institution was obtained. It is a hospital- based descriptive, observational, comparative study done from August 2014 to May 2015.

\section{Inclusion criteria:}

a) All patients with clinical features of anterior urethral stricture.

\section{Exclusion criteria:}

a) Proximal bulbar and bulbo-membranous urethral stricture.

b) History of recent traumatic catheterization.

c) Obliterated urethral stricture.

d) Bladder outlet obstruction or conditions responsible for urinary retention.

e) Active urinary tract infection.

f) Meatal or submeatal stricture.

g) Pan anterior urethral stricture.

h) Patients who did not undergo or gave negative consent for treatment were excluded from the study.

A total of seventy-seven patients (Post Hoc power analysis) satisfying the inclusion criteria were included in the study. Past and present detailed history was taken and physical examination was done. Routine urinalysis followed by uroflowmetry and radiological investigations were done. Written informed consent was obtained from the patients.

All patients were further investigated via RGU and Micturating Cystourethrogram (MCUG) followed 4-6 days later by SUG and SE performed by a separate experienced senior radiologist who was blinded to the RUG report. ${ }^{[3]}$

Standard dynamic RUG and MCUG techniques were used. The patient was placed in supine, 45-degree oblique position and using a 10-12 Fr Foley catheter (in case of meatal stenosis feeding tube was used), $10-15 \mathrm{~mL}$ of contrast medium $(76 \%$ Urografin) was injected into the urethra and spot films were taken. ${ }^{[6,8]}$

Sonourethrography was performed using a 12 Fr Foley catheter through which saline was injected as described by McAninch et al. ${ }^{[2,12]}$ The urethra was screened by a $7.5 \mathrm{MHz}$ linear array transducer through the ventral surface of penis up to bulbo-membranous junction using trans-scrotal and trans-perineal approach. Multiple cross-sectional and longitudinal images were obtained. Stenotic segments were identified as areas of reduced distensibility with saline injection. Cases where the proximal extent of the stricture was not clear, the patient was asked to void with a full bladder, which helped to delineate the proximal extent. Spongiofibrosis appeared as areas of hyperechogenicity.

The following parameters were recorded: site, number, length, and diameter of the stricture, spongiofibrosis, other periurethral pathology (presence of false tracts, filling defects, diverticula, etc.) and complications, if any. The parameters were recorded using an electronic scale for RUG (after correcting for magnification at $100 \%$ magnification) and SUG.

After detailed B-mode sonourethrographic examination, urethra was evaluated by SE (HITACHI HI-VISION PREIRUS) with a linear (L-74M) 5-13 MHz transducer, kept perpendicular to the skin surface. The urethra was then gently compressed with the specific probe and slowly released. The adequacy of the compression was indicated by real-time elastography scale displayed on the screen during scanning. Images were considered optimum when the adequacy criterion for compression was optimally attained. The sonoelastographic pattern (elastogram) of urethral spongiofibrosis after adequate compression was evaluated and categorized into three groups according to the tissue stiffness. Stiffness is depicted in a continuum of colours from red to green to blue designating soft (i.e high strain), intermediate (equal strain) and hard (no strain) tissue. ${ }^{[17,18]}$ The procedure was repeated at least once to avoid spurious results. The strain was calculated as an independent parameter irrespective of ultrasound $\mathrm{B}$ mode or elastogram characteristics. The most representative image obtained with optimal compression factor was stored for further assessment. Other parameters evaluated by SE were same as that of SUG. Stricture length on SE was measured as the length of the colour pattern while depth as vertical extent of the colour pattern.

Stricture length was classified as short $(\leq 15 \mathrm{~mm})$, intermediate $(16-25 \mathrm{~mm})$ and long $(>25 \mathrm{~mm})$ segment. ${ }^{[8]}$ Stricture severity was based on the parameters like degree of luminal narrowing on SUG, colour of mucosa and difficulty of incision according to McAninch et al. ${ }^{[12]}$ as depicted in Table 1. ${ }^{[2,3,5]}$ Spongiofibrosis 
was graded as mild (involving $<1 / 3^{\text {rd }}$ of corpus spongiosum thickness), moderate $(1 / 3-1 / 2)$ and severe $(>1 / 2){ }^{[3]}$

All the cases were managed either by visual internal urethrotomy (VIU) by single author (V.T) or open surgery. During VIU, stricture length was measured by the markings on Sachse' urethrotome sheath while a measuring scale was used at open surgery. Assessment of severity of stricture was done according to the data elicited in Table $1 .{ }^{[3,5]}$

Retrograde urethrography, SUG and SE findings were then independently compared with operative findings. Histopathological assessment of stricture segments for the degree of spongiofibrosis was done on specimens obtained from open surgery cases for validation of intra-operative grading. Haematoxylin and eosin, and Massons' trichome stained slides were used for the evaluation of the same.

\section{Statistical analysis}

Statistical analysis was done using computer software Primer and MS Excel. The qualitative data were expressed in proportions and percentages and the quantitative data as mean and

\section{Table 1. Parameters for assessing severity of}

spongiofibrosis

\begin{tabular}{|lccc} 
SUG & $\begin{array}{c}\text { Operative findings } \\
\text { (degree of luminal, } \\
\text { nal narrowing) }\end{array}$ & $\begin{array}{c}\text { Colour of the } \\
\text { urethral mucosa }\end{array}$ & $\begin{array}{c}\text { Difficulty in } \\
\text { incision }\end{array}$ \\
\hline Mild & $<33 \%$ & Pink & Mild \\
\hline Moderate & $33-50 \%$ & Grey & Moderate \\
\hline Severe & $>50 \%$ & White & Severe \\
\hline SUG: sonourethrography & & \\
\hline
\end{tabular}

standard deviations. The difference in proportion was analysed by using chi- square test and the difference in means were analysed by using Student $\mathrm{T}$ test and one way ANOVA, which were further analysed by post- hoc test (Tukey test). Diagnostic accuracy, sensitivity, specificity and predictive values were calculated for different investigation modalities against the cystoscopic and histopathological findings. Level of significance for tests was determined within $95 \%$ confidence interval $(p<0.05)$.

\section{Results}

The commonest presenting complaint was poor stream of urine (59.4\%, 44 patients) followed by dysuria (23.3\%, 18 patients). Mean age of the patients was $34.67 \pm 0.97$ (range: $21-56$ years). Post- catheterization (42.86\%), idiopathic (28.57\%) and traumatic strictures $(9.09 \%)$ were observed.

Strain pattern of blue and green colour were in equal proportion as evaluated on SUG. Evaluation of 77 patients revealed that $45.5 \%(35 / 77)$ of the patients underwent VIU, $43.0 \%$ (33/77) had open surgery (mainly anastomotic and tube flap substitution urethroplasty) while $11.5 \%$ (9/77) of them were found to have normal urethra on cystoscopy. A total of 82 strictures were demonstrated in 77 male patients, of which 79 strictures were diagnosed on RUG while 74 strictures on SUG and SE compared to 71 intra-operatively detected strictures. Most common stricture site was bulbar region of urethra followed by peno-bulbar region and no significant difference was observed among the imaging modalities in aspect to stricture site localisation.

Mean stricture length measured by RUG, SUG, and SE was $16.37 \pm 10.09 \mathrm{~mm}, 17.6 \pm 10.37 \mathrm{~mm}$ and $22.54 \pm 11.03 \mathrm{~mm}$ respec-

Table 2. Diagnostic accuracy of RUG, SUG and SE regarding stricture length

\begin{tabular}{|c|c|c|c|c|c|c|c|c|c|}
\hline \multirow[b]{2}{*}{ Statistical test } & \multicolumn{3}{|c|}{ RUG } & \multicolumn{3}{|c|}{ SUG } & \multicolumn{3}{|c|}{ SE } \\
\hline & Short & Intermediate & Long & Short & Intermediate & Long & Short & Intermediate & Long \\
\hline Total no. of pts. & 52 & 18 & 9 & 40 & 22 & 12 & 37 & 19 & 18 \\
\hline True positive & 22 & 9 & 9 & 28 & 16 & 12 & 28 & 19 & 18 \\
\hline False positive & 30 & 9 & 0 & 12 & 6 & 0 & 9 & 0 & 0 \\
\hline False negative & 6 & 16 & 9 & 0 & 9 & 6 & 0 & 6 & 0 \\
\hline Sensitivity (\%) & 78.57 & 36 & 50 & 100 & 64 & 66.7 & 100 & 76 & 100 \\
\hline Specificity (\%) & 44.44 & 84.2 & 100 & 77.8 & 89.5 & 100 & 80.43 & 100 & 100 \\
\hline Accuracy (\%) & 85.2 & 69.5 & 89 & 85.4 & 82 & 92.7 & 87.84 & 92 & 100 \\
\hline $\operatorname{PPV}(\%)$ & 42.3 & 50 & 100 & 70 & 72.7 & 100 & 75.68 & 100 & 100 \\
\hline NPV (\%) & 80 & 75 & 87.7 & 100 & 85 & 91.4 & 100 & 89.1 & 100 \\
\hline
\end{tabular}


Table 3. Assessment of spongiofibrosis by SUG and SE together with cystoscopic findings

\begin{tabular}{|c|c|c|c|c|c|c|c|c|c|c|c|}
\hline \multirow{4}{*}{ SUG } & \multicolumn{9}{|c|}{ Cystoscopy } & & \\
\hline & \multicolumn{5}{|c|}{ Colour of the urethral mucosa } & \multicolumn{6}{|c|}{ Difficulty in incision } \\
\hline & \multirow{2}{*}{$\begin{array}{c}\text { Total } \\
\text { n }\end{array}$} & \multicolumn{2}{|c|}{ Grey } & \multicolumn{2}{|c|}{ White } & \multicolumn{2}{|c|}{ Mild } & \multicolumn{2}{|c|}{ Moderate } & \multicolumn{2}{|c|}{ Severe } \\
\hline & & $\bar{n}$ & $\%$ & $\bar{n}$ & $\%$ & $\bar{n}$ & $\%$ & $\mathbf{n}$ & $\%$ & n & $\%$ \\
\hline Number & 34 & 21 & 61.76 & 13 & 38.24 & 15 & 44.12 & 13 & 38.24 & 6 & 17.65 \\
\hline Mild & 14 & 10 & 47.62 & 4 & 30.77 & 6 & 42.86 & 8 & 57.14 & 0 & 0 \\
\hline Moderate & 17 & 11 & 52.38 & 6 & 46.15 & 9 & 52.94 & 5 & 29.41 & 3 & 50.00 \\
\hline Severe & 3 & 0 & 0.00 & 3 & 23.08 & 0 & 0.00 & 0 & 0.00 & 3 & 50.00 \\
\hline $\mathrm{p}$ value & & & $\mathrm{p}=0.065$ & & & & & & $\mathrm{p}=0.449$ & & \\
\hline \multicolumn{12}{|l|}{ SE } \\
\hline Mild & 16 & 12 & 57.14 & 4 & 30.77 & 9 & 60 & 7 & 53.85 & 0 & 0.00 \\
\hline Moderate & 12 & 9 & 42.86 & 3 & 23.08 & 6 & 40 & 3 & 23.08 & 3 & 50.00 \\
\hline Severe & 6 & 0 & 0.00 & 6 & 46.15 & 0 & 0 & 3 & 23.08 & 3 & 50.00 \\
\hline $\mathrm{p}$ value & & & $\mathrm{p}=0.003$ & & & & & & $\mathrm{p}=0.127$ & & \\
\hline
\end{tabular}

Table 4. Assessment of spongiofibrosis by SUG and SE together with histopathology findings

\begin{tabular}{|c|c|c|c|c|c|c|c|}
\hline \multirow[b]{2}{*}{ Spongiofibrosis } & \multirow[b]{2}{*}{ Histopathology } & \multicolumn{2}{|c|}{ Mild } & \multicolumn{2}{|c|}{ Moderate } & \multicolumn{2}{|c|}{ Severe } \\
\hline & & SUG & SE & SUG & SE & SUG & SE \\
\hline Moderate & 9 & 2 & 3 & 6 & 3 & 1 & 3 \\
\hline Severe & 28 & 0 & 0 & 19 & 0 & 9 & 28 \\
\hline Total & 37 & 2 & 3 & 25 & 3 & 10 & 31 \\
\hline Diagnostic accuracy (\%) & & 80.49 & 76.83 & 74.29 & 74.39 & 71.95 & 89.02 \\
\hline $\mathrm{p}$ value & & \multicolumn{2}{|c|}{ NA } & \multicolumn{2}{|c|}{$\mathrm{p}=0.333$} & \multicolumn{2}{|c|}{$\mathrm{p}<0.001$} \\
\hline
\end{tabular}

tively. The mean length measured on sonoelastography was closest to the mean intra-operatively detected stricture length $(21.34 \pm 11.8 \mathrm{~mm})$.

Overall, diagnostic accuracy in predicting stricture length for short, intermediate and long strictures was $85.2 \%, 69.5 \%$ and $89 \%$ by RUG, $85.4 \%, 82.0 \%$ and $92.7 \%$ by SUG and $87.84 \%$, $92 \%$ and $100 \%$ by SE, respectively. Sonoelastography showed higher accuracy in estimating the lengths of intermediate and long segment strictures as compared to other two techniques (Table 2).

Significant association $(\mathrm{p}=0.003)$ between SE findings and colour of the mucosa on cystoscopy was found whereas nonsignificant correlation between SE findings and difficulty in incision was observed. Sonourethrographic findings were not significantly correlated with both colour of the urethral mucosa and difficulty in incision on cystoscopy (Table 3).

Thirty-four patients were managed by open surgery in the form of excisional, and primary anastomosis and substitution urethroplasty e.g. prepucial tube flap. A total of 37 strictures were found during open surgery and assessed histopathologically. Out of these 37 strictures, 28 had severe while 9 had moderate degree of spongiofibrosis. The diagnostic accuracy of SE in predicting spongiofibrosis compared to the gold standard histopathology was $76.83 \%, 74.39 \%$ and $89.02 \%$ for mild, moderate and severe degree of spongiofibrosis whereas SUG has $80.49 \%$, $74.29 \%$ and $71.95 \%$ accuracy for the same pathology, respectively. Significant association was observed for severe degree 

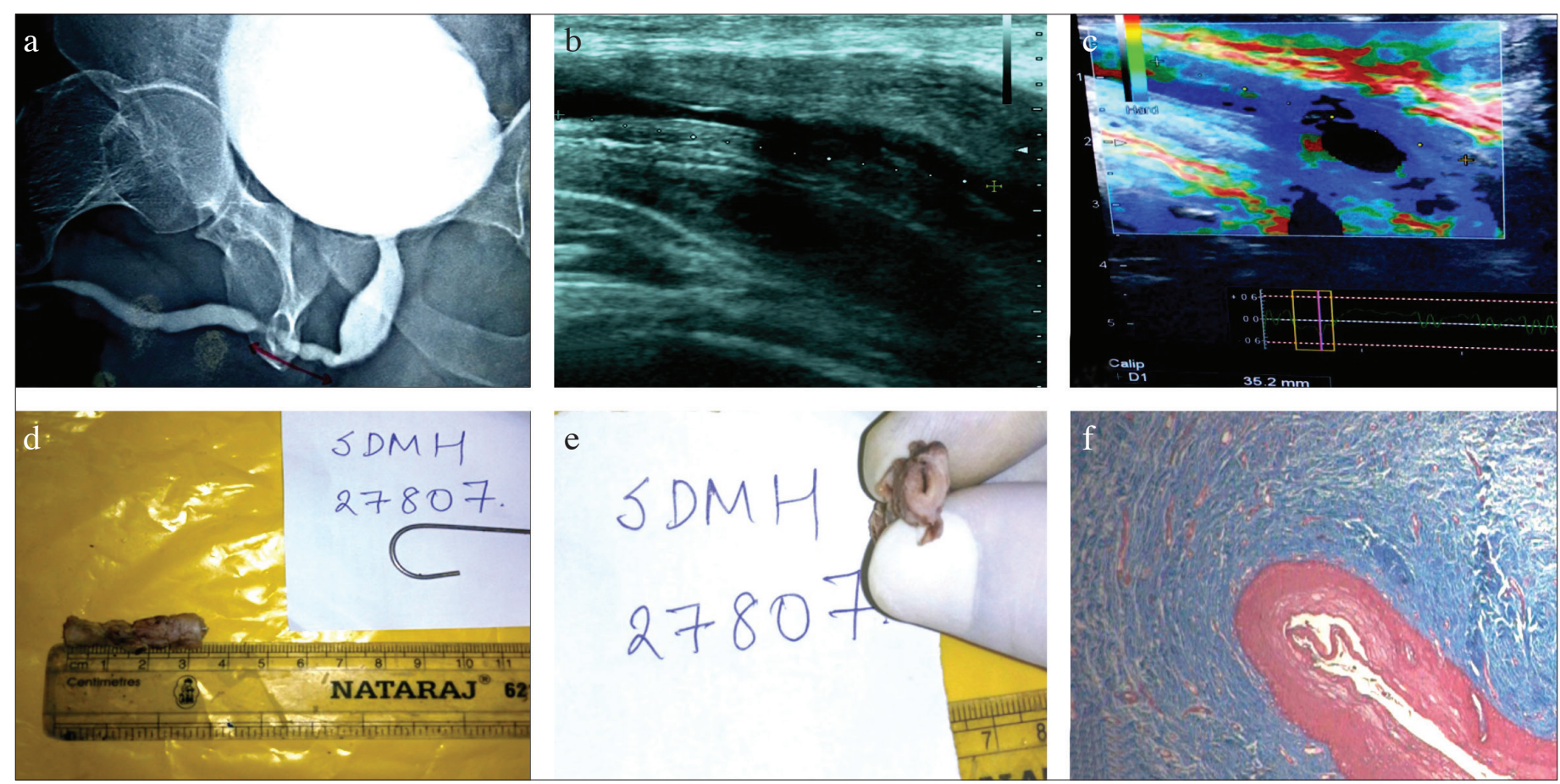

Figure 1. a-f. (a) Retrograde urethrography showing penobulbar urethral stricture segment measuring $23 \mathrm{~mm}$ in length. (b) Sonourethrography showing the same stricture segment measuring $28 \mathrm{~mm}$ in length with moderate degree of fibrosis. (c) Sonoelastography is also showing the same stricture segment with blue pattern, measuring $35 \mathrm{~mm}$ in length and with severe degree of fibrosis. (d, e) Gross specimen of the same urethral stricture segment measuring $33 \mathrm{~mm}$ in length and showing thickened wall. (f) Micrograph depicting severe degree of fibrosis involving full thickness of the wall (X 100, Masson's trichrome stain)

of fibrosis between SE and histopathology findings $(\mathrm{p}<0.001)$ (Table 4).

Associated periurethral pathology such as fistula was diagnosed in $3.80 \%$ of the cases on RUG and $4.05 \%$ on SUG. Complications observed during RUG were fever and intravasation of dye in $5.06 \%$, dysuria in $3.8 \%$, and urethral bleeding in $2.5 \%$ of the cases. No such significant complications were observed during SUG and SE procedures.

\section{Discussion}

Various imaging modalities are available for the evaluation of the urethral stricture, but up to date RUG is the most common and preferred modality despite its well-known limitations and disadvantages like inadequate positioning of the patient and penile traction during contrast injection leading to alteration in stricture length and overlapping, inability to evaluate spongiofibrosis, extravasation and intravasation of dye in cases of forceful injection of contrast material, underestimation of length of anterior especially bulbar urethral stricture ${ }^{[5,6,7]}$, radiation exposure to gonads and contrast allergy in some susceptible patients. ${ }^{[3-6,8,9]}$ To overcome these limitations, an adjuvant technique of sonourethrography was introduced by McAninch et al. ${ }^{[12]}$ in $1988^{[3,5,7,8]}$ which provided better estimation of stricture length $^{[2]}$, delineation of spongiofibrosis and periurethral pathology with an added advantage of no radiation exposure and hypersensivity reaction. Various studies have been conducted for the comparison of both techniques.

Priyadarshi et al.$^{[8]}$ reported an overall sensitivity and accuracy of SUG in predicting stricture length as $95.55 \%$ vs. $97.33 \%$ compared to RUG (77.22\% vs. $85.33 \%)$. They also reported an accuracy of $78 \%, 66 \%$ and $88 \%$ for assessment of mild, moderate and severe spongiofibrosis. Mitterberger et al. ${ }^{[7]}$ found that correlation between RUG and SUG regarding stricture length was stronger for penile compared to bulbar urethra. Various other studies have shown that SUG was better in predicting location, and length of the stricture and it had an advantage of delineating spongiofibrosis and periurethral pathology more accurately. ${ }^{[3-7,9-11]}$ However, echogenicity and mechanical attributes of the tissue do generally not correlate, and therefore proper estimation of tissue stiffness will provide a better idea of the underlying fibrosis. ${ }^{[18,19]}$ Spongiofibrosis is a crucial determinant of surgical outcome and prognosis. Its severity is directly proportional to recurrence of stricture and may dictate management. ${ }^{[2,5,6,18]}$ Nash et al. ${ }^{[20]}$ highlighted a shortcoming of SUG, that it cannot accurately measure the depth of spongiofi- 

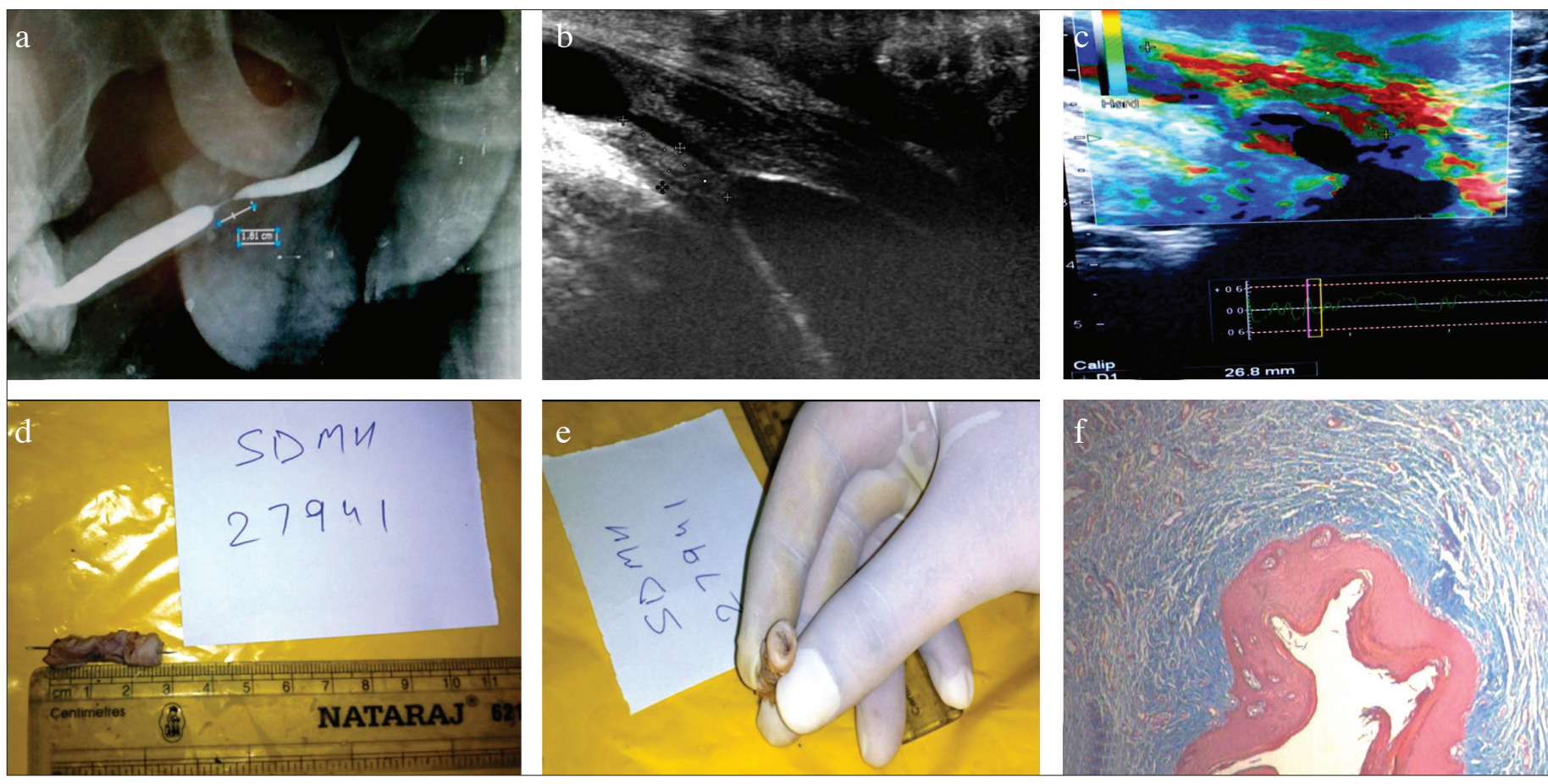

Figure 2. a-f. (a) Retrograde urethrography showing penile urethral stricture segment measuring $18 \mathrm{~mm}$ in length. (b) Sonourethrography showing the same stricture segment measuring $22 \mathrm{~mm}$ in length with mild degree of fibrosis. (c) Sonoelastography also showing the same stricture segment with green pattern, measuring $26 \mathrm{~mm}$ in length and with moderate degree of fibrosis. (d, e) Gross specimen of the same urethral stricture segment measuring $27 \mathrm{~mm}$ in length and showing thickened wall. (f) Micrograph depicting moderate degree of fibrosis (X 100, Masson's trichrome stain)
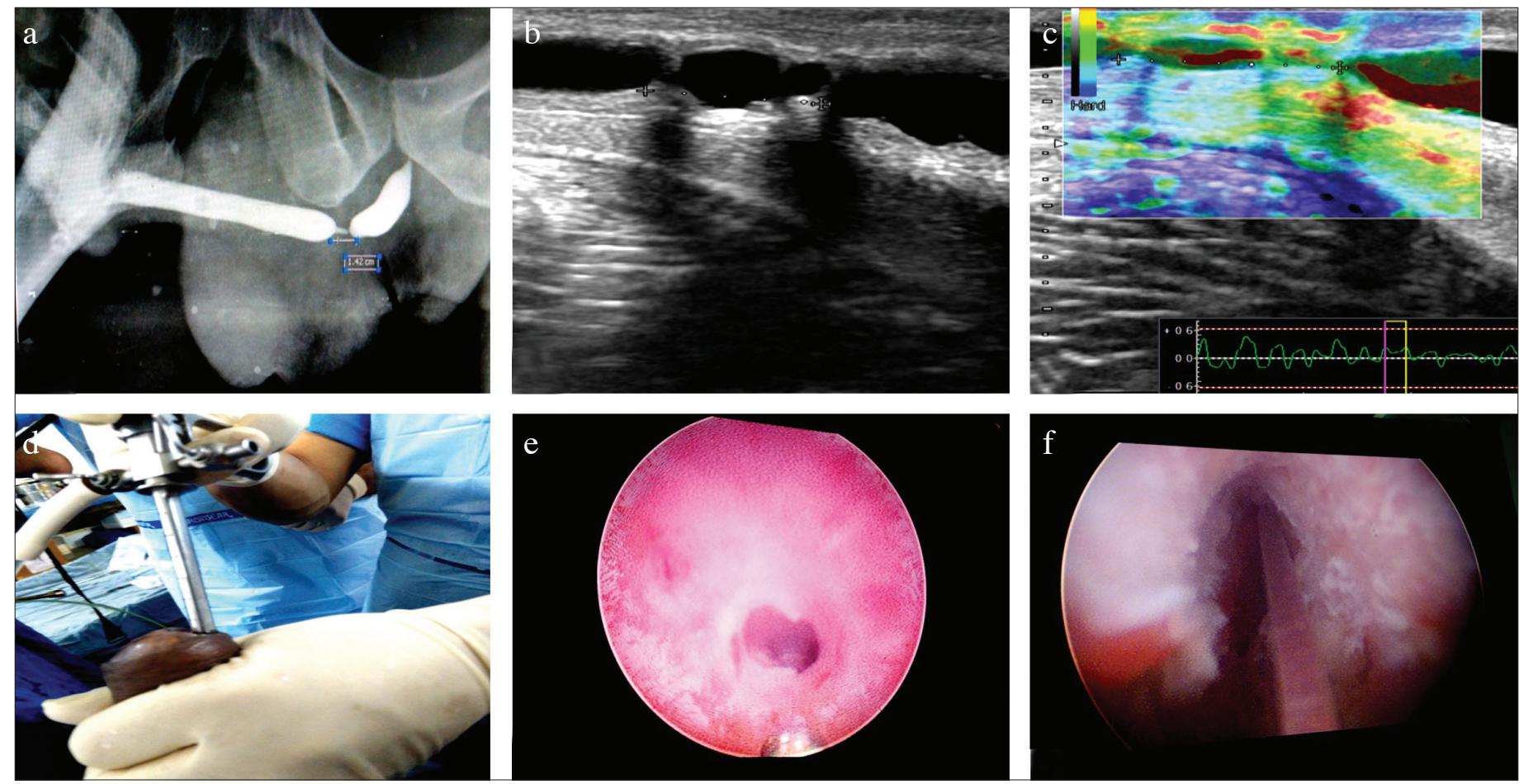

Figure 3. a-f. (a) Retrograde urethrography showing bulbar urethral stricture segment measuring $14 \mathrm{~mm}$ in length. (b) Sonourethrography showing the same stricture segment measuring $18 \mathrm{~mm}$ in length with mild degree of fibrosis. (c) Sonoelastography also showing the same stricture segment with blue pattern, measuring $22 \mathrm{~mm}$ in length and with moderate degree of fibrosis. (d) On cystoscopy, the same stricture segment measured $25 \mathrm{~mm}$ in length, (e) had white coloured mucosa and (f) severe difficulty in incision was observed during visual internal urethrotomy 
brosis when compared with histopathological assessment which was in agreement with other studies ${ }^{[3,5,9,20]}$ RUG utilizes intraluminal opacification, therefore it provides minimal information about direct assessment of periurethral pathology. ${ }^{[1,3]}$

Sonoelastography also known as virtual or electronic palpation is a novel technique used for measurement of tissue stiffness. ${ }^{[21]}$ Compared to manual palpation which has a historical importance in physical examination, ${ }^{[22]} \mathrm{SE}$ has an advantage of evaluating deeper lesions and furthermore it is semi-quantifiable. The basic principle of this technique is that an abnormal tissue (i.e. the one affected by fibrosis, inflammation or neoplastic process) is stiffer than the normal tissue. It is this property of SE, which we have especially utilized (estimating longitudinal extent and depth of spongiofibrotic segment) in the evaluation of anterior urethral strictures; as spongiofibrosis is one of the most important determinant in planning treatment and prognosis. ${ }^{[2,17,21]}$ To our best knowledge, this is the first study assessing the role of sonoelastography in the evaluation of anterior urethral strictures, which could be a promising potential target for this upcoming imaging technology.

Strain elastography is defined as a technique of imaging tissue elasticity or stiffness by measuring spatial rate of tissue displacement in response to certain amount of pressure applied on the tissue. Strain is defined as a relative change in shape or size of an object due to externally applied forces and it is expressed as change in length during compression divided by length before compression. Stress is force per unit area. Stiffness of tissue is calculated via Young's modulus, defined as $\mathrm{E}=$ stress/strain. As most machines measure strain and not Young's modulus, direct quantification is not possible. ${ }^{[15,17]}$ The strain map is called elastogram. SE is performed in a split screen mode with the conventional B-mode image on the right, and the elastogram on the left side. ${ }^{[17,18]} \mathrm{SE}$ measures tissue stiffness both qualitatively, and semi-quantitavely, ${ }^{[15,17]}$ in the form of strain pattern and strain ratio, respectively. Strain images show the relative stiffness of the lesion compared with the adjacent tissue stiffness. ${ }^{[15,23]}$ Strain pattern is depicted in continuum of colours as described earlier in this study. However, colour standardization is yet not present and some SE machines follow an inverse colour scale different from others. ${ }^{[17]}$

In our study, SE correlated better in estimation of stricture location, length and degree of spongiofibrosis when compared with intra-operative and histopathological findings.

Overall diagnostic accuracy, for estimation of stricture location by SE, SUG and RUG was $92.68 \%, 79 \%$ and $80.48 \%$ respectively while for stricture length SE, SUG, and had diagnosti accuracies as $91.54,78.87$, and $43.66 \%$, respectively. SE yielded an accuracy of $87.3 \%$ for estimation of spongiofibrosis compared to $48 \%$ of SUG.

Estimation of periurethral pathology like presence of false tracts on SE was comparable to SUG but better than RUG. Estimation of stricture length and depth is of vital importance as it forms the basis of description among cases for endoscopic management (short segment strictures) and open surgery (intermediate and long segment requiring end to end anastomosis and prepucial tube replacement). ${ }^{[2]}$ The starting and end point of the stricture length were estimated from the point where the narrowing had started to begin. SE displayed better visualization of stricture length and by virtue of strain pattern we were able to visualize extension of the length compared with B-mode and RUG. Degree of spongiofibrosis was also delineated better by SE as it displayed more accurate estimation of extent of depth. Depth of spongiofibrosis observed by SE was compared with SUG measurements and validated by intra-operative and histopathological assessment as depicted in Figures 1, 2, and 3. Complications during SE procedure were none to minimal ( 1 case). Choudhary et al. ${ }^{[3]}$ reported pain, urethral bleeding and contrast medium intravasation during RUG procedure.

Our study showed that SE provided more accurate estimation of urethral stricture evaluation parameters in comparison to RUG and SUG and it correlated excellently with intra-operative and histopathological findings. It best estimated spongiofibrosis which is an important prognostic factor for stricture recurrence. Therefore, it should be routinely used as an adjuvant to RUG and SUG.

Ethics Committee Approval: Ethics committee approval was received for this study from the ethics committee of Sawai Man Singh Medical College and Hospital (No. Dated: 1689/MC/EC/2015).

Informed Consent: Written informed consent was obtained from patients who participated in this study.

Peer-review: Externally peer-reviewed.

Author Contributions: Concept - S.M.T., V.T., S.S.Y., U.J., N.A., N.V.; Design - S.M.T., S.S.Y., S.P., N.V.; Definition of Content S.M.T., V.T., U.J., N.A., N.V.;Data Collection and/or Processing - S.M.T., V.T., S.S.Y., U.J., S.P., N.A., N.V.; Statistical Analysis S.M.T., V.T., S.S.Y., U.J.; Literature Search - S.M.T., U.J., S.P., N.A., N.V.; Clinical Studies - S.M.T., S.S.Y., S.P., N.A.; Experimental Studies - S.M.T., V.T., S.P., N.V.; Writing Manuscript - S.M.T., V.T., U.J., N.A.; Critical Review - S.M.T., S.S.Y., S.P., N.V.; Other - S.M.T., V.T., S.S.Y., U.J., S.P., N.A., N.V.

Acknowledgements: The authors wish to thanks Dr. Divya Pursnani for her help in grammatical proof reading of the write-up of study and Dr. Rashmi Sharma for help in statistical analysis of the study. 
Conflict of Interest: No conflict of interest was declared by the authors.

Financial Disclosure: The authors declared that this study has received no financial support.

\section{References}

1. Maciejewski C, Rourke K. Imaging of urethral stricture disease. Transl Androl Urol 2015;491:2-9.

2. Dahiya N, Menias CO, Siegel CL. Imaging of the male urethra. In: Brandes SB, Morey AF, editors. Advanced Male urethral and genital reconstructive surgery, 2nd ed. New York: Humana press; 2014. p. 51-68. [CrossRef]

3. Choudhary S, Singh P, Sundar E, Kumar S, Sahai A. A comparison of sonourethrography and retrograde urethrography in evaluation of anterior urethral strictures. Clin Radiol 2004;59:736-42. [CrossRef]

4. El-ghar MA, Osman Y, Elbaz E, Refiae H, Diasty TE. MR urethrogram versus combined retrograde urethrogram and sonourethrography in diagnosis of urethral stricture. Eur J Radiol 2010;74:193-8. [CrossRef]

5. Gupta N, Dubey D, Mandhani A, Shrivastava A, Kapoor R, Kumar A. Urethral stricture assessment: a prospective study evaluating urethral ultrasonography and conventional radiological studies. BJU Int 2006;98:149-53. [CrossRef]

6. Ravikumar BR, Tejus C, Madappa KM, Prashant D, Dhayanand GS. A comparative study of ascending urethrogram and sono-urethrogram in the evaluation of stricture urethra. IBJU 2015;41:388-92.

7. Mitterberger M, Christian G, Pinggera GM, Bartsch G, Strasser H, Pallwein L, et al. Gray scale and color doppler sonography with extended field of view technique for the diagnostic evaluation of anterior urethral strictures. J Urol 2007;177:992-7. [CrossRef]

8. Priyadarshi V, Singh M, Kumar V, Tiwari R, Gupta SK, Sehgal $\mathrm{N}$. The role of sonourethrography in the evaluation of anterior urethral strictures: A correlation with retrograde urethrography. Uro Today Int J 2012;5:1-6. [CrossRef]

9. Mandal SK, Bhattacharyya SK, Mandal A, Deoghuria D, Mandal PK. Sonourethrography in the evaluation of anterior urethral stricture: correlation with retrograde urethrography in male. Int $\mathbf{J}$ Pharm Biomed Res 2012;3:77-80.
10. Hatgaonkar A, Pendharkar P. Sonourethrography in evaluation of abnormalities of anterior male urethra. IOSR-JDMS 2014;13:53-9. [CrossRef]

11. Khan N, Modishi HM, Tsatsi LD, Khaloon A, Seogone A. Comparison of sonourethrography and retrograde urethrography in the evaluation of anterior urethral strictures. S Afr J Rad 2004;8:6-9. [CrossRef]

12. McAninch JW, Laing FC, Jeffery Jr RB. Sonourethrography in the evaluation of urethral strictures: a preliminary report. J Urol 1988;139:294-7.

13. Dudea SM, Jid CB. Ultrasound elastography in thyroid disease. Med Ultrason 2015;17:74-96.

14. Kapoor A, Kapoor A, Mahajan G. Real-time Elastography in acute appendicitis. J Ultrasound Med 2010;29:871-7.

15. Mousa AE, Aboelatta M, Zalata K. Combined sonoelastographic scoring and strain ratio in evaluation of breast masses. EJRNM 2012;43:647-56. [CrossRef]

16. Lyshchik A, Higashi T, Asato R, Tanaka S, Ito J, Hiraoka M, et al. Cervical lymph node metastases: diagnosis at sonoelastography-initial experience. Radiology 2007;243:258-67. [CrossRef]

17. Carlsen JF, Ewertsen C, Önn L, Nielsen MB. Strain elastography ultrasound: An overview with emphasis on breast cancer diagnosis. Diagnostics 2013;3:117-25. [CrossRef]

18. Gheorghe L, Lacob S, Gheorghe C. Real-time Sonoelastographya new application in the field of liver disease. J Gastrointestin Liver Dis 2008;17:469-74.

19. Ophir J, Kallel F, Varghese T, Konofagou E, Kaisar Alam SKS, Krouskop T, et al. Elastography. Optical and Acoustical Imaging of Biological Media 2001;2:1193-212. [CrossRef]

20. Nash PA, McAninch JW, Bruce JE, Hanks DK. Sonourethrography in the evaluation of anterior urethral strictures. J Urol 1995;154:72-6. [CrossRef]

21. Ruchala M, Szczepanek-Parulska E, Zybek A, Moczko J, Czarnywojtek A, Kaminski G, et al. The role of sonoelastography in acute, subacute and chronic thyroiditis: a novel application of the method. Eur J Endocrinol 2012;166:425-32. [CrossRef]

22. Wells PNT, Liang HD. Medical ultrasound: imaging of soft tissue strain and elasticity. JR Soc Interface 2011;8:1521-49. [CrossRef]

23. Barr RG, Nakashima K, Amy D, Cosgrove D, Farrokh A, Schafer F, et al. WFUMB guidelines and recommendations for clinical use of ultrasound elastography: Part 2: breast. Ultrasound Med Biol 2015;41:1148-60. [CrossRef] 